\title{
Endocrine, Cardiovascular, and Behavioral Responses to Clonidine in Patients with Panic Disorder
}

\author{
James L. Abelson, Debra Glitz, Oliver G. Cameron, \\ Myung A. Lee, Michael Bronzo, and George C. Curtis
}

We examined adrenergic regulation in patients with panic disorder by challenging 10 patients and 14 age-matched and sex-matched controls with intravenous infusions of clonidine hydrochloride $(2 \mu \mathrm{g} / \mathrm{kg})$, an alpha $\mathrm{a}_{2}$-adrenoreceptor agonist. Growth hormone, 3-methoxy-4-hydroxyphenylglycol (MHPG), blood pressure, heart rate, and behavioral (ansiety, sedation) responses were monitored. The data replicated the previously reported finding of blunted growth hormone $(\mathrm{GH})$ responses to clonidine in patients with panic disorder. Reported abnormalities in MHPG, cardiovascular, and behavioral responses of panic patients to clonidine infusion were not replicated. The robustly blunted $\mathrm{GH}$ response to clonidine in panic patients supports the adrenergic dysregulation hypothesis of panic disorder, but alternative interpretations of this finding are available and further study is needed.

\section{Introduction}

Blunted growth hormone $(\mathrm{GH})$ responses to clonidine infusion (Charney and Heninger 1986; Nutt 1989; Uhde ct al 1986) provide the most compelling evidence to date of an adrenergic abnormality in patients with panic disorder. However, one attempt to replicate this finding has failed (Schittecatte et al 1988). Clonidine has also been reported to produce enhanced decreases in blood pressure and 3-methoxy-4-hydroxyphenylglycol (MHPG) and reduced sedation in panic patients compared to controls (Charney and Heninger 1986; Nutt 1989); but these findings have not been uniformally replicated (Uhde et al 1989). The effect of clonidine on anxiety has also differed asross studies (Charney and Heninger 1986; Uhde et al 1989). In an attempt to replicate the blunted GH reponses to clonidine in panic disorder, and to clarify the status of cardiovascular, MHPG, and sedation/anxiety responses, we conducted clonidine infusions in additional groups of panic patients and normal control subjects.

From the Department of Psychiatry. University of Michigan, Ann Arbor, Michigan.

Address reprint requests to James L. Abelson. M.D., Ph.D., University of Michigan Medical Center, 1500 E. Medical Center Drive, Ann Artor, Ml 48109-0840.

Received July 29, 1991; March 29, 1992. Presently at 'Wayne State University (DG); 'Case Western Reserve University (MAL); ${ }^{3}$ University of California at San Diego
(MB). 


\section{Methods}

Fourteen control subjects (mean age $=33.4 \pm 10.0$ years, 7 men and 7 women) and 10 panic patients (mean age $=35.6 \pm 5.5$ years, 5 men) were studied. Patients met DSM-III criteria for panic disorder, had experienced at least three panic attacks in the 3 weeks prior to study, were not currently depressed or abusing substances, and had no history of depression prior to onset of their panic disorder. No control subject had a history of any DSM-III Axis I disorder. Medical history, physical examination, and screening laboratory studies ensured that subjects were healthy and drug free for at least 2 weeks before testing. Females were studied within the first 10 days of the menstrual cycle, were not menopausal, and were not taking oral contraceptives. Subjects with regular alcohol consumption equivalent to, or greater than, an average of four cans of beer per week were excluded. All subjects gave written informed consent.

Procedures, assays, and analyses are described in detail elsewhere (Abelson et al 1991). Briefly, subjects were studied on two mornings within an 8-day period following a night in a clinical research center. They were given single-blind infusions of placebo ( $10 \mathrm{ml}$ of normal saline) on day 1 and clonidine hydrochloride $(2 \mu \mathrm{g} / \mathrm{kg}$ in $10 \mathrm{ml}$ normal saline over $5 \mathrm{~min}$ ) on day 2 (mean dose infused $=124 \mu \mathrm{g}$ ). Blood samples were taken for GH and MHPG assay $15 \mathrm{~min}$ and immediately before infusion, and at 15, 30, 45, $60,90,120$, and $180 \mathrm{~min}$ thereafter. Systolic (SBP) and diastolic (DBP) blood pressure, heart rate (HR), and symptom ratings (visual analog scales and Spielberger State Anxiety Scale) were obtained at each time point. Subjects were not permitted to sleep during the study. Subjective ratings of stress and anxiety for the 6 months prior to the study were obtained on day 1. Subjects completed the trait portion of the Spielberger State-Trait Anxiety Inventory (STAI) (Spielberger et al 1969) and a Carroll Rating Scale for Depression (Carroll et al 1981) on each study day. Sleep quality was rated on a 3-point scale (restful, fair, or poor). General level of exercise was rated on a 4-point scale ( $1=$ no regular exercise, 4 = aerobic exercise $\geqslant 3$ times/week). Plasma GH was measured by double-antibody radioimmunoassay (Glick et al 1963) and plasma MHPG by high-performance liquid chromatography with coulometric detection (Hariharan et al 1989). Hormone data was log-transformed to approximate normal distributions. Two subjects (1 patient, 1 control) were excluded from GH analyses due to elevated baseline levels. One control subject was excluded from some GH and MHPG analyses due to a missed blood sample at the $45-\mathrm{min}$ postinfusion time point.

\section{Results}

Clonidine strongly stimulated release of $\mathrm{GH}$ (repeated measures analysis of variance; main effect of drug: $F[1,19]=12.5, p<0.005$; drug-by-time interaction: $F[8,152]=$ $15.42, n<0.001$ ), but did so less robustly in panic patients (diagnosis-by-drug, $F[1,19]$ $=7.27, p<0.05$, and diagnosis-by-time, $F[8,152]=3.10, p<0.005$, interactions were both significant, though the three-way interaction was not, $F[8,152]=1.52, p=$ $0.15)$. The blunted response of the panic patients was most evident at 30, 45, and 60 min after infusion (see Figure 1). Six of nine $(67 \%)$ panic patients were GH nonresponders (GH peak minus last baseline before infusion of less than $5 \mathrm{ng} / \mathrm{ml}$ ), coinpared to 4 of 12 control subjects $(33 \%)$.

Of the six panic patients who had blunted GH responses to clonidine, four were 

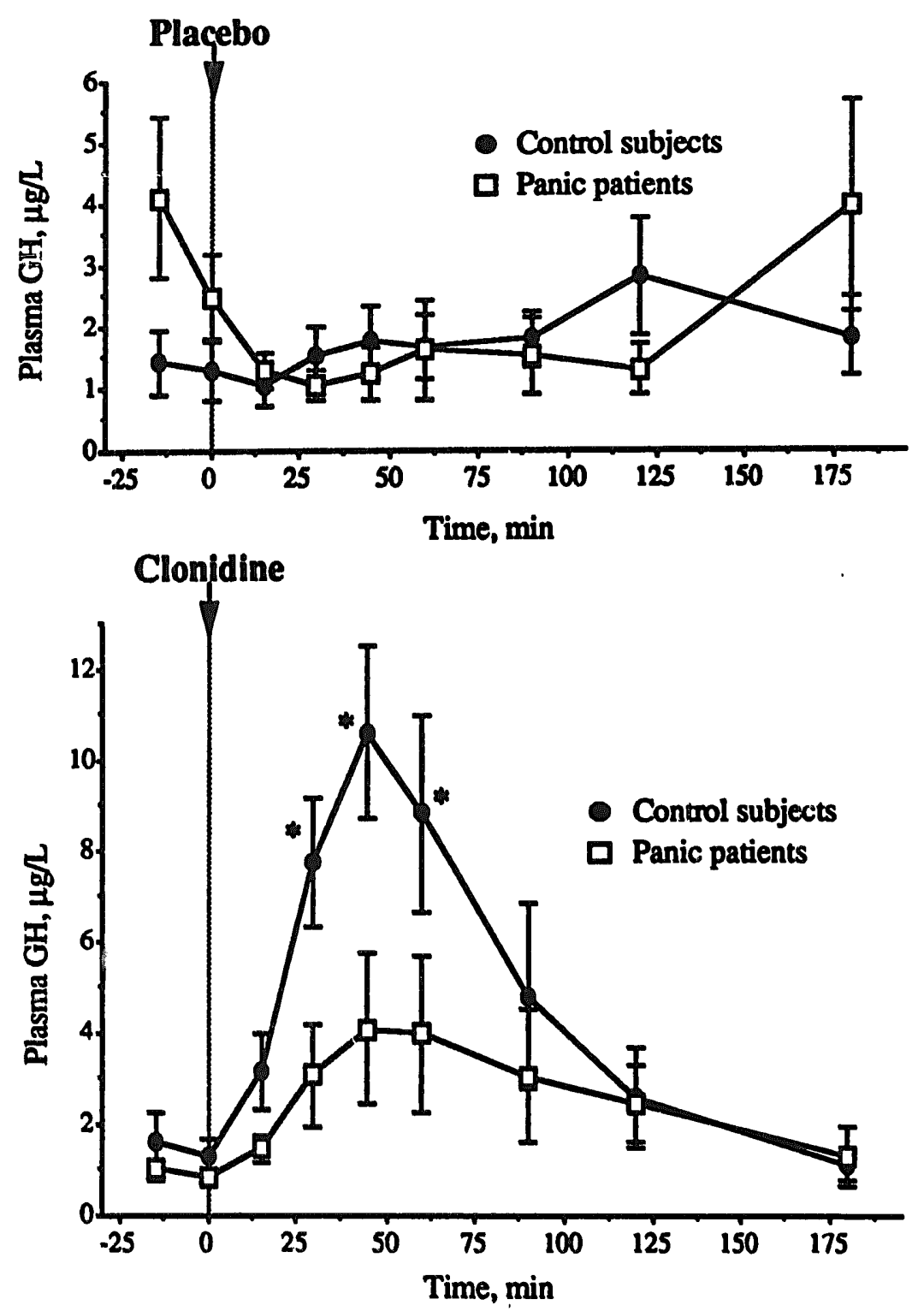

Figure 1. Growth hormone (GH) responses to placebo (upper graph) and clonidine (lower graph) in patients with panic disorder and normal subjects. Group means and SEMs are plotted at each sampling point (15 and $0.5 \mathrm{~min}$ before, and $15,30,45,60,90,120$, and $180 \mathrm{~min}$ after infusion). The groups differed significantly $(t$-tests, $p<0.05$ ) at the time points marked with asterisks.

evaluated using structured interviews with reliable data about treatment histories and comorbid diagnoses. None of these four patients had any history of prior exposure to tricyclic antidepressant medication. None had any history of a major depressive episode. Only one of the four met criteria for generalized anxiety disorder or dysthymia (she met criteria for both).

$\mathrm{GH}$ responses to clonidine (mean of the $\mathrm{GH}$ level at 30,45 , and $60 \mathrm{~min}$ after infusion minus the GH level just prior to infusion) were not significantly correlated with Carroll depression scores, STAI trait anxiety, weight, exercise levels, or sleep quality. Higher 

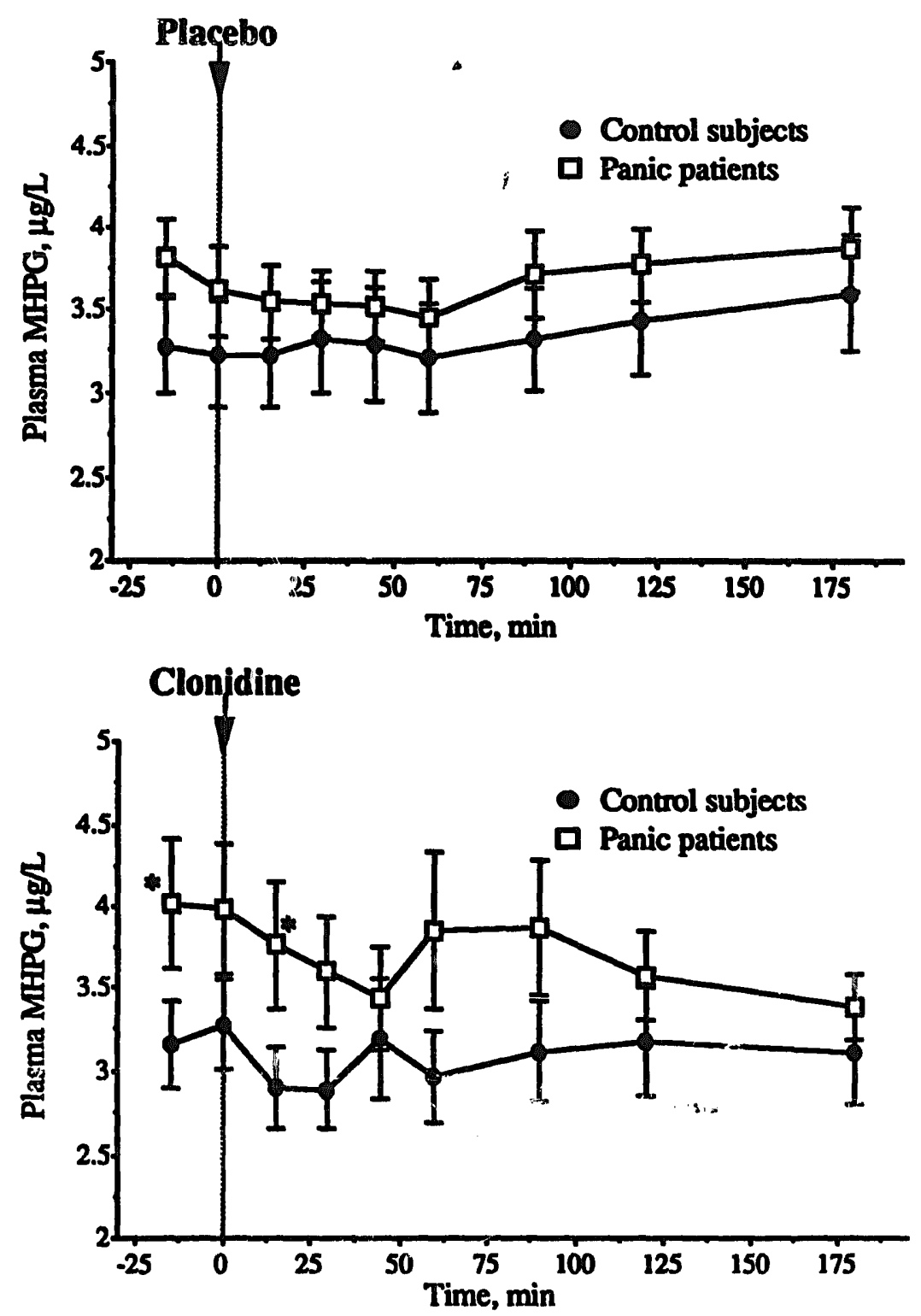

Figure 2. Plasma 3-methoxy-4-hydroxyphenoiglycol (MHPG) responses to placebo (upper graph) and clonidine (lower graph) in patients with panic disorder and normal subjects. Group means and SEMs are plotted at each sarnpling point (15 and $0.5 \mathrm{~min}$ before, and 15, 30, 45, 60, 90, 120 , and $180 \mathrm{~min}$ after infusion). The groups differed significantly ( $t$ tests, $p<0.05$ ) at the time points marked with asterisks.

levels of stress and anxiety for the 6 months prior to study (self-rated on a 10-point scale; $1=$ none, $10=$ worst ever) were associated with more blunted $\mathrm{GH}$ responses $(r=$ $-0.48, p<0.05$ and $r=-0.54, p<0.05$, respectively).

Clonidine lowered MHPG levels (see Figure 2; main effect of time: $F[8,176]=3.23$, $p<0.005$; drug-by-time interaction: $F[8,176]=3.78, p<0.001)$ but no interactions involving diagnosis were significant. Patients had higher MHPG levels than controls throughout the procedures, but the main effect of diagnosis was not significant $(F[1,22]=2.18, p=0.15)$. The difference between groups in maximal MHPG change 
Table 1. Maximum Decreases on Cardiovascular Measures After Clonidine Infusion ${ }^{a}$

\begin{tabular}{lcr}
\hline & Patients with PD & Healthy controls \\
\hline Systolic blood pressure, $\mathrm{mmHg}$ & $19.4 \pm 4.2$ & $17.9 \pm 4.1$ \\
Diastolic blood pressure, $\mathrm{mmHg}$ & $15.3 \pm 7.5$ & $13.6 \pm 4.6$ \\
Heart rate, beats/min & $6.1 \pm 4.3$ & $5.5 \pm 6.5$ \\
\hline
\end{tabular}

${ }^{a}$ Values are mean $\pm \mathrm{SD}$.

${ }^{b} \mathrm{PD}$ indicates panic disorder.

after clonidine (lowest level after infusion minus mean baseline level) was nut significant $(t[22]=1.61, p=0.12$; controls' mean maximal decline $\pm \mathrm{SD}=0.5 \pm 0.3 \mu \mathrm{g} / \mathrm{L}$, patients' $=0.9 \pm 0.6 \mu \mathrm{g} / \mathrm{L})$. The small group difference became even smaller after declines following placebo were subtracted $(t[22]=0.90, p=0.38$; controls' mean $=$ $0.3 \pm 0.04 \mu \mathrm{g} / \mathrm{L}$, patients' $=0.5 \pm 0.6 \mu \mathrm{g} / \mathrm{L}$ ). Because group differences were in the expected direction but not significant and our sample size was small, we pursued additional analyses. Examination of Figure 2 suggests that at 45 min after infusion the patient group's MHPG reached a nadir, whereas the control group's MHPG at this time point was near baseline. However, the placebo-corrected MHPG decline from baseline to 45 min post-infusion did not differ between groups $(t[22]=1.27, p=0.22$; controls' mean $=0.2 \pm 0.5 \mu \mathrm{g} / \mathrm{L}$, patients' $=0.5 \pm 0.6 \mu \mathrm{g} / \mathrm{L})$. We found no relationship between the $\mathrm{GH}$ response to clonidine and the maximal MHPG change $(r=0.22, p=0.32)$.

Clonidine reduceis SBP and DBP in patients and controls (main effects of drug: $F[1,22]=117.66, p<0.0001 ; F[1,22]=40.94, p<0.001$; main effects of time: $F[8,176]=27.07, p<0.001 ; F[8,176]=7.04, p<0.001$; drug-by-time interactions: $F[8,176]=19.78, p<0.001 ; F[8,176]=12.01, p<0.001)$. None of the effects involving diagnosis were significant. Decline in HR following clonidine was reflected in a significant drug-by-time interaction $(F[8,176]=3.49, p<0.001)$. There was a main eî́rect of diagnosis $\{F[1,22]=6.93, p<0.05)$; but no interactions involving diagnosis were significant. Mean HR for patients over the entire monitoring period was 67.2 beats per min, compared to 59.2 beats per min for controls. To maximize our chance of detecting group differences in cardiovascular responses to clonidine we also compared groups on maximum responses, calculated by subtracting each subject's lowest value after infusion from their baseline. The groups did not differ on any of these variables $(t[22]=0.87$, $p=0.40 ; t[22]=0.65, p=0.53 ;$ and $t[22]=0.29, p=0.77$, respectively; see Table 1).

Clonidine produced substantial sedation in patients and controls (main effects of drug: $F[1,22]=26.48, p<0.001$, and time: $F[8,176]=11.61, p<0.001$; drug-by-time interaction: $F[8,176]=14.16, p<0.001$ ). Patients had elevated ratings of drowsiness throughout the study (main effects of diagnosis: $F[1,22]=4.75, p<0.05$ ); but no interactions involving diagnosis were significant. The difference between groups in their peak drowsiness response to clonidine was not significant $(t[22]=0.88, p=0.39$; controls' mean increase in drowsiness rating from baseline to post-clonidine peak = $4.3 \pm 3.1$, patients' $=5.8 \pm 1.8$ ) and was opposite in direction to that predicted by prior research (increased rather than blunted sedative response).

State anxiety was higher in patients throughout the study (main effect of diagnosis: $F[1,19]=39.83, p<0.001$ ), fell over the course of each day in both groups (main effect of time: $F[8,152]=7.21, p<0.001$ ), and fell to a greater extent in patients than 
controls (diagnosis-by-time interaction: $F[8,152]=2.20, p<0.05$ ). However, the degree of anxiolysis did not differ between placebo and clonidine days (no significant effects involving drug) and was related to baseline levels $(r=0.55, p<0.01)$. Similar results were obtained from analyses of analogue ratings of "worry."

\section{Discussion}

Four of five published studies have now demonstrated significantly smaller GH responses to clonidine in panic patients compared to controls (Charney and Heninger 1986; Nutt 1989; Uhde et al 1986). Approximately 37 of the 56 panic patients studied to date (66\%) have had blunted responses (less than a $5 \mathrm{ng} / \mathrm{ml}$ increase in $\mathbf{~} \mathrm{H}$ after clonidine infusion), compared to approximately 18 of 57 control subjects $(32 \%)\left(\chi^{2}=13.5, p<0.001\right)$. The single failure to replicate (Schittecatte et al 1988) involved only seven subjects, and blunting was absent only in the female subjects, in whom birth control pills or menstrualcycle phase can obscure blunting. It is unlikely that the $\mathrm{GH}$ blunting seen in anxiety disorders is an epiphenomenon of tricyclic exposure as four of our six panic patients and all $r f$ our six generalized anxiety disorder patients (Abelson et al 1991) with blunted responses had no significant prior exposure to tricyclic antidepressants.

Blunted GH response to clonidine is thought to reflect subsensitivity of post-synaptic alpha $_{2}$-adrenoreceptors (Siever et al 1982), but its implications are complicated by a report of blunted GH responses to GH-releasing hormone (GHRH) in panic (Rapaport et al 1989). Blunted GH responses to both clonidine and GHRH could result from chronic excessive adrenergic drive to the hypothalamus but abnormalities within the pituitary or in other neurotransmitter systems could also account for the findings. Studies that combine the use of GHRH and multiple adrenergic probes in the same subjects are needed.

Only one placebo-controlled study of panic patients has demonstrated an enhanced decline in MHPG following clonidine infusion (Charney and Heninger 1986). A single replication did not include a control infusion and was complicated by elevated baseline MHPG in the patients (Nutt 1989). Our group difference were in the expected direction but were not significant. This failure to replicate could be caused by the small sample size. However, it appeared in our data that any potential differences between patients and controls in MHPG was present on both placebo and clonidine days and may not reflect differential responsivity to clonidine per se.

Ours is the third of four studies to show no difference between patients and controls in SBP response to clonidine (Charney and Heninger 1986; Uhde et al 1989). The one report of an exaggerated decline in patients used a method of data analysis that should be more sensitive to briefer, less stable blood-pressure changes. However, we could not detect group differences or even trends using either this or our preferred more conservative method. Enhanced declines in DBP have been demonstrated in two studies (Charney and Heninger 1986; Nutt 1989); but our data provide the second failure to replicate this finding (Uhde et al 1989). Studies are consistent in showing some "tonic" elevation of HR in panic patients but normal HR responses to clonidine.

The two prior placebo-controlled studies examining acute anxiolytic activity of clonidirie in panic patients produced conflicting results (Charney and Heninger 1986; Uhde et al 1989). Our data and those of Charney and Heninger (1986) indicate that anxiety declines equally following placebo and clonidine infusions and patient-control differences are likely secondary to elevated baseline anxiety in patients. The group differences in 
anxiolysis reported by Uhde et al (1989) could be due to substantially higher baseline anxiety in the patients. The greater decline in anxiety following clonidine rather than placebo, within their patient group, may also be due to baseline differences as the patients appear to have had higher baseline state anxiety scores on clonidine day than on placebo diuy. Given baseline effects on anxiety responses and the likely sensitivity of panic patients' baseline anxiety to experimental procedures, available data does not clearly demonstrate true pharmacological differences between panic patients and controls in their anxiolytic responses to clonidine infusion. Other experimental designs will be needed to conclusively show that clonidine possesses acute anxiolytic activity in panic patients. Our data, like those of Uhde et al (1989), did not replicate a blunted sedative response to clonidine in panic patients (Charney and Heninger 1986; Nutt 1989). The elevated mean drowsiness ratings in our patients, compared to controls, make it unlikely that failure to replicate was due to insufficient power.

The blunted GH response to clonidine in patients with panic disorder appears highly reproducible. Other reported "abnormal" responses to clonidine in panic (i.e., enhanced declines in MHPG, blood pressure and anxiety, and reduced sedation) appear less replicable, because each has now failed to replicate at least twice. Small samples and differences in procedures could account for some of the inconsistencies. Variable baseline effects and other uncontrolled factors (e.g., weight and exercise levels) may also play a role. We cannot conclude from our small sample that panic patients do not have some abnormalities in MHPG, blood pressure, sedative, or anxiety responses to clonidine; but the inconsistencies in these variables across multiple studies make the reproducibility of the GH abnorinality all the more striking. GH blunting likely represents a true physiological abnormulity in the regulation of the hypothalamic-pituitary-somatotrophic axis in panic patients. Whether it reflects subsensitivity of postsynaptic alpha ${ }_{2}$-adrenoreceptors remains to be proven. It does represent an unequivocal finding that requires further exploration.

This research was supported in part by NIMH grant ROIMH42736 and in part by Clinical Research Center grant MOIRR00042. Clonidine was generously supplied by Boehringer-Ingelheim Pharmaceuticals.

\section{References}

Abelson JL, Glitz D, Cameron OG, Lee MA, Bronzo M, Curtis GC (1991): Blunted growth hormone response to clonidine in patients with generalized anxiety disorder. Arch Gen Psychiatry 48:157-162.

Carroll BJ, Feinberg M, Smouse PE, Rawson SG, Greden JF (1981): The Carroll Rating Scale for Depression, I: Development, reliability and validation. Br J Psychiatry 138:194-200.

Charney DS, Heninger GR (1986): Abnormal regulation of noradrenergic function in panic disorder. Arch Gen Psychiatry 43:1042-1054.

Glick R, Roth J, Yalow R, Berson S (1963): Immunoassay of human growth hormone in plasma. Nature 199:784.

Hariharan M, VanNoord T, Cameron OG, Curtis GC, Ostrow DG (1989): Free 3-methoxy-4hydroxyphenylglycol determined in plasma by liquid chromatography with coulometric detection. Clin Chem 35:202-205.

Nutt DJ (1989): Altered central alpha2-adrenoreceptor sensitivity in panic disorder. Arch Gen Psychiatry 46:165-169.

Rapaport MH, Risch SC, Gillin JC, Golshan S, Janowsky DS (1989): Blunted growth hormone 
response to peripheral infusion of human growth hormone-releasing factor in patients with panic disorder. Am J Psychiatry 146:92-95.

Schittecatte M, Charles G, Depauw Y, Mesters P, Wilmotte J (1988): Growth hormone response to clonidine in panic disorder patients. Psychiatry Res 23:147-151.

Siever L, Lhde T, Silberman E, et al (1982): Growth hormone response to clonidine as a probe of noradrenergic receptor responsiveness in affective disorder patients and controls. Psychiatry Res 6:171-183.

Spielberger CD, Gorsuch RL, Lushene RE (1969): STAI Manual for the State-Trait Anxiety Inventory. Palo Alto, CA: Consulting Psychologists Press.

Uhde TW, Vittone BJ, Siever LJ, Kaye WH, Post RM (1986): Blunted growth hormone response to clonidine in panic disorder patients. Biol Psychiatry 21:1081-1085.

Uhde TW, Stein MB, Vittone BJ, et al (1989): Behavioral and physiological effects of short-term and long-term administration of clonidine in panic disorder. Arch Gen Psychiatry 46:170-177. 